\title{
The hospital environment and its microbial burden: challenges and solutions
}

loana Chirca*,1

${ }^{1}$ University Hospital, University Healthcare System, Augusta, GA 30901, USA

*Author for correspondence: chirca.ioana@gmail.com

\begin{abstract}
“There has been increasing interest for alternative technologies such as self-disinfecting surfaces. Metallic silver and copper have intrinsic antimicrobial activity and are continuously active when built into high touch surfaces"
\end{abstract}

First draft submitted: 13 May 2019; Accepted for publication: 12 July 2019; Published online: 30 August 2019

Keywords: bioburden $\bullet$ environmental contamination $\bullet$ hydrogen peroxide $\bullet$ copper surfaces $\bullet$ disinfectants $\bullet$ UV light

"The ultimate measure of a man is not where he stands in moments of comfort and convenience, but where he stands at times of challenge and controversy" [1]. As appropriate as this was for the civil rights movement, it continues to apply to other areas, such as the healthcare environment. Affected by multiple outbreaks and with looming threats of multidrug-resistant infections, the healthcare systems continue to focus on understanding the epidemiology of hospital infections. An important part of this is the role of environmental contamination in transmission of nosocomial pathogens and development of hospital-acquired infections (HAI). We aim to describe the challenges presented by the bioburden in the hospital environment and the currently available solutions.

One of the first few questions that require answers is whether there is a baseline level of contamination on environmental surfaces; what is the latency of organisms on these surfaces? Subsequently, can environmental cleaning and disinfection can reduce contamination of these surfaces. Perhaps the more important question is whether presence of organisms on environmental surfaces increases the risk for HAI and whether a specific level of contamination exists with a greater impact on transmission to patients.

Several studies showed that patients shed microorganisms to their surrounding environment. This is higher in those infected versus those colonized and near-patient surfaces such as bedrails, bedside tables, call buttons have a higher bioburden [2]. One study showed the mean microbial bioburden was higher $\left(2700 \mathrm{CFU} / 100 \mathrm{~cm}^{2}\right)$ in routinely cleaned rooms versus terminally cleaned rooms $\left(353 \mathrm{CFU} / 100 \mathrm{~cm}^{2}\right)$ [3]. Transmission of potential pathogens inside the hospital is complex and involves contaminated hands of healthcare workers, contaminated equipment or contamination of the room from a prior patient. One study identified methicillin-resistant Staphylococcus aureus (MRSA) isolates in hospital rooms of patients without known MRSA colonization status, although less often than those with known colonization status [4]. Pathogenic microorganisms persist in the hospital environment from hours to months, depending on factors such as location, number, biofilm formation, intrinsic resistance of organisms to various cleaning products as well as local conditions. This has been well documented for S. aureus, Enterococcus to include vancomycin-resistant strains (VRE), Clostridium difficile, Pseudomonas aeruginosa, Acinetobacter species [5]. More recently, emerging pathogens such as Candida auris have shown survival for at least 7 days in a moist environment [6] and the activity of antiseptics and disinfectants on this pathogen is largely unknown and under investigation [7]. Most studies involving interventions to improve cleaning and disinfection practices were usually quasi-experimental in design. The majority were able to show decreased environmental contamination, with variable effect on multidrug resistant organism (MDRO) acquisition (from decreased to no effect on acquisition); notably, some $C$. difficile studies showed no effect on incidence of $C$. difficile infection from enhanced environmental cleaning and disinfectant interventions [8] although, others have shown significant decrease in the incidence of CDI $[16,20]$. 
Potential pathogens are able to transfer from the environment to the patient and can become involved in the development of a HAI. One prospective multicenter cohort study showed bacterial transfer events in $18.5 \%$ of patient encounters and these occurred early in the admission [9]. Another determined that an environmental reservoir played a major role in a VRE outbreak [10]. One study was able to show that environmental levels of Enterococcus and Escherichia coli were positively correlated with infection incidences [11]. Prospectively followed intensive care unit patients had their environment sampled weekly and revealed that the risk of HAI significantly increased as the microbial burden increased [12].

Another important challenge is assessing effectiveness of environmental cleaning and disinfection. While the Centers for Disease Control and Prevention (CDC) does not recommend routine microbiological sampling, in addition to visual inspection and checklist, various novel techniques have been employed, with excellent results; for example: fluorescent marker method, adenosine triphosphate bioluminescence assay and ultraviolet powder [13,14].

With this knowledge, the $\mathrm{CDC}$ has recommended guidelines for hand hygiene and personal protective equipment use as well as for cleaning and disinfecting the environment of care [23].

Routine and terminal environmental cleaning and disinfection is typically bundled with other practices to control the environmental microbial burden. The CDC recommends the use of disinfectants such as chlorine, alcohol, aldehydes, quaternary ammonium compounds, iodophors, peracetic acid, hydrogen peroxide and phenolics. Cotton-based cloths and mop heads may be more attractive from a cost perspective as they can be washed multiple times at high temperatures. It has been found that ultra-microfiber cloths and other microfiber products have the potential of enhanced cleaning [15], although study results are discordant, and their use is still controversial. These products and disinfectants are often used either alone or in combination, along with educational interventions, increased volume of disinfectants, increased surveillance and other techniques to terminate outbreaks or simply improve the bioburden. Another challenge is with the dependency of infection control practices meant for enhancing and optimizing environmental cleaning and disinfection, on human factors. The inability to comply with the bundle processes in a consistent fashion may limit the benefits. This factor led to the development of novel disinfecting agents and techniques, using no-touch delivery systems such as hydrogen peroxide vapor (HPV), UV light decontamination units, steam cleaning devices, ozone generators, multijet cold-plasma units, copper-surfaced objects and antimicrobial surfaces. It is to be noted that these technologies are meant to enhance decontamination of the hospital environment but are more effective when employed in conjunction with physical removal of bioburden.

A recent meta-analysis has found that ultraviolet light no-touch disinfection technology may be effective in reducing CDI rates and VRE infection rates but has found no effect on rates of MRSA or gram-negative MDRO infections [16]. Multiple other studies have shown effective bioburden reduction using UV light units for terminal decontamination, for example a reduction in vegetative organisms by $99.9 \%$ at $15 \mathrm{~min}$ and a reduction in $C$. difficile spores by $99.8 \%$ at 50 min was observed [17]. Additionally, MRSA-positive samples were decreased from 20.3 to $0.5 \%$ in this same study. The UV light technology has some limitations related to variable antimicrobial effect depending on the location and orientation of objects and surfaces relative to the UV light unit, with decreased activity in remote and shadowed areas [18], but multivector ultraviolet systems with shadowless delivery and pulse xenon ultraviolet technologies are being developed. It is important to mention that emerging fungal pathogens such as $C$. auris are significantly less susceptible to UV light than other organisms. One practical challenge is the lack of uniform efficacy standards across different UV light devices, hence the difficulty to compare effectiveness.

Terminal cleaning with hydrogen peroxide via aerosolized or vapor delivery technologies has been evaluated as effective in decreasing the burden of potentially pathogenic bacteria such as MRSA, P. aeruginosa, Acinetobacter baumanii and $C$. difficile in various settings and there is also evidence for decreasing HAI, specifically CDI $[19,20]$. As with UV light, the hydrogen peroxide delivery technologies vary and consequently, so do the results. Additionally, supplementary training of environmental services personnel is required to comply with strict safety measures during the use of hydrogen peroxide delivery systems.

A prospective observational study comparing HPV and UV light showed reduction in bacterial contamination including spores for both devices but concluded that HPV is significantly more effective, mostly due to decreased antimicrobial effect in the shadowed areas for UV light devices [21].

There has been increasing interest for alternative technologies such as self-disinfecting surfaces. Metallic silver and copper have intrinsic antimicrobial activity and are continuously active when built into high touch surfaces. It has been shown consistently that copper coating or copper alloy-surfaced materials reduce environmental colonization with a sustained effect. In addition, a reduction in the composite outcome measure of HAI and certain MDRO colonization was noted for patients treated in Intensive Care Unit rooms with copper-coated objects [12]. Other 
surfaces such as antimicrobial polymers in solution or on surfaces have also shown encouraging results. For example, photocatalytic antimicrobial coatings containing titanium dioxide have shown reduced bioburden from high-risk surfaces in the hospital [22]. However, a definite link with a decrease in HAIs has not yet been determined. In addition, concerns about the durability of this technology, possible resistance and allergenic properties have been raised.

The microbial burden present in the hospital environment appears to significantly affect colonization and subsequent infection statuses of patients, playing an important role in the development of HAI. Although imperfect, various strategies and bundled strategies have been shown to improve the cleaning and disinfection of the hospital environment, subsequently decreasing the incidence of certain HAI, important and current challenges in environmental infection control remain. These stem from the human dependency factors, difficulties with measuring the degree of cleanliness, differences in delivery systems for the same technology, the cost effectiveness of certain strategies, emerging pathogens and resistance within a wide variation of hospital practices and healthcare delivery systems. Fortunately, there is mounting evidence to support healthcare environment cleaning practices that will hopefully mitigate some of the unwanted effects of increasingly resistant pathogens with devastating potential.

\section{Financial \& competing interest's disclosure}

The authors have no relevant affiliations or financial involvement with any organization or entity with a financial interest in or financial conflict with the subject matter or materials discussed in the manuscript. This includes employment, consultancies, honoraria, stock ownership or options, expert testimony, grants or patents received or pending, or royalties.

No writing assistance was utilized in the production of this manuscript.

\section{References}

1. Martin Luther King Jr. Strength to Love. Harper \& Row, NY, USA (1963).

2. Dancer SJ, White LF, Lamb J et al. Measuring the effect of enhanced cleaning in a UK hospital: a prospective cross-over study. BMC Med. 7, 28 (2009).

3. Shams A, Rose L, Edwards J et al. Assessment of the overall and multidrug-resistant organism bioburden on environmental surfaces in healthcare facilities. Infect. Control Hosp. Epidemiol. 37(12), 1426-1432 (2016).

4. Villamaria F, Berlanga G, Liao I et al. Comparison of environmental MRSA levels on high-touch surfaces in contact isolation and noncontact isolation patient rooms. Infect. Control Hosp. Epidemiol. 36(12), 1472-1475 (2015).

5. Otter JA, Yezli S, Salkeld JA et al. Evidence that contaminated surfaces contribute to the transmission of hospital pathogens and an overview of strategies to address contaminated surfaces in hospital settings. Am. J. Infect. Control 41, S6-S11 (2013).

6. Piedrahita CT, Cadnum JL, Jencson AL et al. Environmental surfaces in healthcare facilities are a potential source for transmission of Candida auris and other Candida species. Infect. Control Hosp. Epidemiol. 38(9), 1107-1109 (2017).

7. Rutala WA, Kanamori H, Gergen MF et al. Susceptibility of Candida auris and Candida albicans to 21 germicides used in healthcare facilities. Infect. Control Hosp. Epidemiol. 40(3), 380-382 (2019).

8. Ray A, Deshpande A, Fertelli D et al. A multicenter randomized trial to determine the effect of an environmental disinfection intervention on the incidence of healthcare-associated clostridium difficile infection. Infect. Control Hosp. Epidemiol. 38(7), 777-783 (2017).

9. Chen L, Knelson L, Gergen M et al. A prospective study of transmission of multidrug-resistant organisms (MDROs) between environmental sites and hospitalized patients - the TransFER study. Infect. Control Hosp. Epidemiol. 40(1), 47-52 (2019).

10. Lee A, White E, Monahan L et al. Defining the role of the environment in the emergence and persistence of vanA vancomycin-resistant enterococcus (VRE) in an intensive care unit: a molecular epidemiological study. Infect. Control Hosp. Epidemiol. 39(6), 668-675 (2018).

11. Lesho E, Carling P, Hosford E et al. Relationships among cleaning, environmental DNA, and healthcare-associated infections in a new evidence-based design hospital. Infect. Control Hosp. Epidemiol. 36(10), 1130-1138 (2015).

12. Salgado CD, Sepkowitz KA, John JF et al. Copper surfaces reduce the rate of healthcare acquired infections in the intensive care unit. Infect. Control Hosp. Epidemiol. 34, 479-486 (2013).

13. Hung IC, Chang HY, Cheng A et al. Application of a fluorescent marker with quantitative bioburden methods to assist cleanliness. Infect. Control Hosp. Epidemiol. 39, 1296-1300 (2018).

14. Munoz-Price LS, Ariza-Heredia E, Adams S et al. Use of UV powder for surveillance to improve environmental cleaning. Infect. Control Hosp. Epidemiol. 32(3), 283285 (2011).

15. Rutala WA, Gergen MF, Weber DJ. Microbiologic evaluation of microfiber mops for surface disinfection. Am. J. Infect. Control. 35(9), 569-573 (2007).

16. Marra A, Schweizer M, Edmond M. No-touch disinfection methods to decrease multidrug-resistant organism infections: a systematic review and meta-analysis. Infect. Control Hosp. Epidemiol. 39(1), 20-31 (2018). 
17. Boyce J, Havill N, Moore B. Terminal decontamination of patient rooms using an automated mobile UV light unit. Infect. Control Hosp. Epidemiol. 32(8), 737-742 (2011).

18. Boyce J, Farrel P, Towle D et al. Impact of Room Location on UV-C irradiance and UV-C dosage and antimicrobial effect delivered by a mobile UV-C light device. Infect. Control Hosp. Epidemiol. 37(6), 667-672 (2016).

19. Blazejewski C, Wallet F, Rouze A et al. Efficiency of hydrogen peroxide and improving disinfection of ICU rooms. Crit. Care 19(1), 30 (2015).

20. Boyce J, Havill N, Otter J et al. Impact of hydrogen peroxide vapor room decontamination on clostridium diffcile environmental contamination and transmission in a healthcare setting. Infect. Control Hosp. Epidemiol. 29(8), 723-729 (2008).

21. Havill N, Moore B, Boyce J. Comparison of the microbiological efficacy of hydrogen peroxide vapor and ultraviolet light processes for room decontamination. Infect. Control Hosp. Epidemiol. 33(5), 507-512 (2012).

22. Reid M, Whatley V, Spooner $\mathrm{E}$ et al. How does a photocatalytic antimicrobial coating affect environmental bioburden in hospitals? Infect. Control Hosp. Epidemiol. 39(4), 398-404 (2018).

23. CDC. Guideline for disinfection and sterilization in healthcare facilities (2008). www.cdc.gov/hicpac/pdf/guidelines/Disinfection_Nov_2008_2008.pdf 